\title{
AMELIORATION OF ACETAMINOPHEN-INDUCED HEPATOTOXICITY USING SOLANUM TORVUM SW. FRUIT EXTRACT IN WISTAR RATS
}

\author{
UMERA BEGAM AK ${ }^{1 *}$, SENTHILKUMAR $\mathbf{R}^{2}$, SIRAJUDEEN J ${ }^{3}$
}

${ }^{1}$ Department of Biochemistry, Holy Cross College (Affiliated to Bharathidasan University), Tiruchirappalli, Tamil Nadu, India. ${ }^{2}$ Department of Biochemistry, Periyar E.V.R. College (Affiliated to Bharathidasan University), Tiruchirappalli, Tamil Nadu, India. ${ }^{3}$ Department of Chemistry, Jamal Mohammed College (Affiliated to Bharathidasan University), Tiruchirappalli, Tamil Nadu, India.

Email: umera150176@gmail.com

Received: 02 September 2019, Revised and Accepted: 15 November 2019

\section{ABSTRACT}

Objective: Ayurveda and Chinese pharmacopeia have highlighted the traditional medicinal uses of Solanum torvum Sw. The fruits are ethnomedical used in the treatment of liver and spleen enlargement, cough, and also used as a hematopoietic, antimicrobial, and analgesic agent. In the present study, the amelioration of acetaminophen (APAP)-induced hepatotoxicity of the aqueous extract of S. torvum Sw. fruits is evaluated.

Methods: The hepatoprotective activity of the fruit extract against APAP insult was evaluated by assessing it is in vivo antioxidants status, membranebound adenosine triphosphatases (ATPases), and tricarboxylic acid (TCA) cycle marker enzymes and also through histopathological studies of the liver.

Results: Administration of the aqueous fruit extract of the plant caused a significant increase in the in vivo antioxidant status as evident from the reduction in lipid peroxidation caused by APAP and improvement in the mitochondrial membrane stability which is proved from the activity of membrane-bound ATPases and TCA cycle marker enzymes. Histological studies also supported the fact that the plant extract proved to revive the architecture of the toxin damaged liver tissues in par with silymarin. The chemical pathological changes were consistent with histopathological observations suggesting marked hepatoprotective effect of the aqueous extract of $S$. torvum.

Conclusion: The results showed that the extract of $S$. torvum Sw. fruits has hepatoprotective potential which may be due to the antioxidant activity of its phytoconstituents, especially flavonoids, alkaloids, phenolics, etc.

Keywords: Hepatoprotective, Liver, Paracetamol, Acetaminophen, Solanum torvum, Antioxidant, Adenosine triphosphatases, Tricarboxylic acid marker enzymes.

(C) 2020 The Authors. Published by Innovare Academic Sciences Pvt Ltd. This is an open access article under the CC BY license (http://creativecommons. org/licenses/by/4. 0/) DOI: http://dx.doi.org/10.22159/ajpcr.2020.v13i1.35564

\section{INTRODUCTION}

Liver, one of the largest organs in human body, is the chief site for intense metabolism and excretion. The liver injury caused by drugs is a major health problem that challenges not only the health care professionals but also the pharmaceutical industry and drug regulatory agencies. Some of the drugs are directly injurious to liver; some others are transformed by liver into chemicals that can be injurious to liver. When drugs injure the liver and disrupt its normal functions, symptoms, and signs of liver diseases develop [1]. More than $50 \%$ of the acute liver failure is accounted for by liver injury caused by drugs, including hepatotoxicity caused by overdose of acetaminophen (APAP). During the detoxification of APAP, reactive oxygen species (ROS) (N-acetyl-p-benzoquinone imine [NAPQI]) are generated which cause oxidative stress leading to hepatic damage. In situations of increased free radical generation, the reinforcement of endogenous antioxidants through intake of dietary antioxidants may be of particular importance in attenuating the cumulative effects of oxidatively damaged molecules. Valuable phytoproducts are yielded by medicinal plants which are useful in the treatment of various diseases. Plants are the rich source of active ingredients for health care products, with many blockbuster drugs being directly or indirectly derived from plants. However, many high value plant-derived natural products remain undiscovered or unexplored for their pharmacological activity [2].

Solanum torvum Sw. is a member of the family Solanaceae and its fruits are commonly called as "night berries" which are used as a common vegetable by South Indian people. It is a prickly, tomentose, erect shrub, 1.5-3 m high, leaves having no prickles, white bell-shaped flowers, and lobed fruits seated on the calyx. It is a common plant found throughout the Indian subcontinent. The fruits are useful for treating liver and spleen enlargement, cough and also used as a hematopoietic, antimicrobial, and analgesic agent. The antiviral and antiulcerogenic property of leaf extract has been reported [3]. The extracts of Sundaikkai fruit coat exhibited significant antibacterial activity against pathogenic bacteria when compared to standard antibiotics and hence acted as natural antibiotic. It is intensively used worldwide in the traditional medicine as poison antidote and for the treatment of fever, wounds, tooth decay, reproductive problems, and arterial hypertension. In this study, we report the hepatoprotective activity of these fruits against APAP-induced liver injury to provide the scientific basis for its use in traditional medicine.

\section{METHODS}

\section{Plants collection}

The $S$. torvum Sw. fruits were procured from Kallanai area in the month of November to January. The plant was identified and authenticated by the Rapinat Herbarium (Ref. No. U002), St. Joseph's College, Tiruchirappalli, Tamil Nadu, India. The air-dried plant material was pulverized with electric grinding machine into minute pieces. A suitable quantity of air-dried and crushed plant powder was weighed and extracted with various solvents in the order of increasing polarity using Soxhlet extraction apparatus (for $6 \mathrm{~h}$ ). The residue was dried at $105^{\circ} \mathrm{C}$ to get a constant weight. 


\section{Animals}

Adult male albino rats of Wistar strain weighing around 200-220 g obtained from the animal house. All the experiments were carried out according to the guidelines of the CPCSEA, New Delhi, India, and approved by the Institutional Animal Ethical Committee Approval No.: 171/SASTRA/IAEC/RPP (2012).

\section{Animal treatment}

For the optimum dose fixation, the aqueous plantextract was administered at different dosages orally for different time intervals. The dosage and the duration of the dosage which give the maximum hepatoprotective activity were chosen for further studies. To study the hepatoprotective activity of the plant extract, the animals were randomized and divided into four groups of six animals each. Group 1 served as normal control and received the sterile distilled water $(10 \mathrm{ml} / \mathrm{kg}$, p.o.) for 7 days. Group 2 is the disease control rats which were administered with APAP at a dose of $600 \mathrm{mg} / \mathrm{kg}$ b.w. orally on the $7^{\text {th }}$ day. Group 3 rats were administered with the optimum dose of the aqueous extract of the plant drug ( $400 \mathrm{mg} / \mathrm{kg}$ body weight p.o.) for 7 days and with APAP on the $7^{\text {th }}$ day $1 \mathrm{~h}$ after pre-treatment with the plant drug and Group 4 rats were treated with silymarin at a dose of $50 \mathrm{mg} / \mathrm{kg}$ b.w. orally for 7 days and were given APAP as above. The extract/silymarin was administered by oral gavages $1 \mathrm{~h}$ before APAP administration [4]. Twenty-four hours after paracetamol administration, animals were sacrificed and blood was collected by retro-orbital puncture.

\section{Assay of in vivo antioxidant profile}

The antioxidant status which is altered in hepatotoxicity is evaluated by assessing the in vivo antioxidant profile in experimental animals which includes estimation of lipid peroxides (LPOs), superoxide dismutase (SOD) catalase, reduced glutathione (GSH), GSH peroxidase (GPx), and GSH-S-transferase (GST) by standard procedures.

Assay of membrane-bound adenosine triphosphatases (ATPases) A very good indicator of APAP toxicity is the mitochondrial dysfunction which can be evaluated by the assay of the membrane-bound ATPases, namely sodium-potassium $\left(\mathrm{Na}^{+} / \mathrm{K}^{+}\right)$, calcium $\left(\mathrm{Ca}^{2+}\right)$, and magnesium $\left(\mathrm{Mg}^{2+}\right)$ ATPases.

\section{Mitochondrial marker enzymes (tricarboxylic acid [TCA] cycle enzymes)}

To determine the mitochondrial damage caused due to lipid peroxidation which in turn was induced by oxidative stress in APAP hepatotoxicity, the TCA cycle enzymes such as isocitrate dehydrogenase (ICDH), succinate dehydrogenase (SDH), and malate dehydrogenases (MDHs) which are located in the inner mitochondrial membrane were estimated by various standard procedures.

\section{Statistical analysis}

The values are expressed as mean and standard error (SE) of the mean. Multiple comparisons of the significant ANOVA were performed by Duncan's Multiple Comparison test. Statistical analysis was performed using SPSS for Windows version 17.0. Data were given in the form of arithmetical mean \pm SE. Differences between groups were evaluated by one way ANOVA according to $\mathrm{p}<0.05$ and post hoc Duncan test.

\section{RESULTS AND DISCUSSION}

\section{Assay of in vivo antioxidant profile}

Fig. 1a,b and c represents the antioxidant status of the control and experimental group of rats. LPO levels are found to be markedly increased $\left({ }^{a} \mathrm{p}<0.01\right)$ and the level of enzymatic antioxidants (SOD, CAT, GPx, and GST) and nonenzymatic antioxidant; GSH is decreased in the liver tissues of APAP-induced rats (Group II) when compared to normal rats. Highly significant reduction in the antioxidant status is observed in rats induced with APAP when compared with normal control rats. These adverse changes are reversed to near normal and an improvement in antioxidant status is noticed in rats treated with the aqueous extract of S. torvum Sw. fruits $\left({ }^{\mathrm{b}} \mathrm{p}<0.01\right)$ before the toxic insult of APAP which is comparable with the standard drug (silymarin)-treated group $\left({ }^{\mathrm{b}} \mathrm{p}<0.01\right)$.
The primary defense against oxidative stress in the tissue rests with antioxidants such as SOD, CAT, GPx, GST, and GSH [5]. APAP exposure produced a marked oxidative impact as reflected by elevated LPO, measured in terms of malondialdehyde (MDA) level in the hepatic tissue when compared to normal control. Lipid peroxidation is a marker of cellular oxidative damage initiated by ROS [6]. The decrease in SOD, CAT, GPx, and GST enzyme activities and a decrease in GSH reductase may indicate the toxic effects of ROS produced by APAP. The hydrogen peroxide and superoxide anions are produced during metabolic activation of APAP in the cytochrome P450 system and from mitochondria during APAP intoxication and the generation of ROS appeared as an early event which preceded intracellular GSH depletion and cell damage in APAP hepatotoxicity [7]. Treatment with aqueous extract of $S$. torvum significantly reverted these changes which may be due to its anti-lipid peroxidative and antioxidant effect. This suggests that the fruit extract can reduce ROS that may lessen the oxidative damage to the hepatocytes and improve the activities of the liver antioxidant enzymes, thus protecting the liver from APAPinduced damage. Furthermore, the possible mechanism could be by the stimulation of hepatic regeneration through an improved synthesis of protein or accelerated detoxification and excretion [6]. The protective effect of the plant extract against APAP may also be related to polyphenolic compounds, terpenoids, alkaloids, coumarin, phytosterols, etc. [8]. Flavonoids are proved to be potent inhibitors of enhanced spontaneous production of both MDA and conjugated dienes and are able to inhibit lipid peroxidation [9].

\section{Membrane-bound ATPases}

The activities of the membrane-bound ATPases are presented in Fig. 2 . The activities of the membrane-bound ATPases are significantly lowered in the disease control groups $\left({ }^{a} \mathrm{p}<0.01\right)$ when compared to normal control group. The plant drug-treated animals showed a marked increase in the activity which is found to be statistically significant when compared to the disease control groups $\left({ }^{b} p<0.01\right)$ same as that of the standard drug silymarin.

As the cell membranes are very susceptible and sensitive to free radical attack, the enhanced abnormal LPO inhibit the activities of ATPases. Hence, elevated lipid peroxidation rate and lowered antioxidant status may be responsible for the observed decreased activities of ATPases in APAP-induced rats [10]. Modulations in $\mathrm{Ca}^{2+}$ levels in the intracellular compartment play a role in cell death. Studies made by Papadimitrious et al. [11] have shown that mitochondrial $\mathrm{Ca}^{2+}$ handling is crucial in various stages leading to cell death due to $\mathrm{Ca}^{2+}$ overload. Hence, the observed decrease in the enzyme activity may be due to $\mathrm{Ca}^{2+}$ overload also. $\mathrm{Ca}^{2+}-$ ATPase inhibition may also be due to the decreased level of GSH. Inhibited activity of $\mathrm{Ca}^{2+}$ - ATPase is reported on exposure to activated oxygen and on depletion of thiol status [12]. The restoration of impairment in the activities of ATPases to near normal in rats pre-treated with S. torvum Sw. fruit extract can be attributed to the potent membranestabilizing effect of $S$. torvum Sw. which will normalize the function of membrane-bound ATPases and other membrane-bound enzymes which are deranged due to oxidative stress leading to increased lipid peroxidation. This antiperoxidative effect may be due to the presence of phenolic and flavonoid compounds which has the potential to restore the deranged activity of membrane-bound ATPases caused by oxidative stress [13].

\section{TCA cycle marker enzymes}

The levels of TCA cycle enzymes are illustrated in Fig. 3a and b. Activities of all the TCA cycle enzymes are significantly $\left({ }^{a} \mathrm{p}<0.01\right)$ decreased in APAP-induced liver damaged group compared to normal control rats. The rats pre-treated with silymarin significantly $\left({ }^{\mathrm{b}} \mathrm{p}<0.01\right)$ restored the levels when compared with liver damaged groups. The pre-treatment with aqueous extract of $S$. torvum Sw. fruits also significantly $\left({ }^{\mathrm{b}} \mathrm{p}<0.01\right)$ restored the levels of $\mathrm{ICDH}, \mathrm{MDH}$ and $\mathrm{SDH}$ when compared to APAPtreated rats (Fig. $3 a$ and $b$ ). 


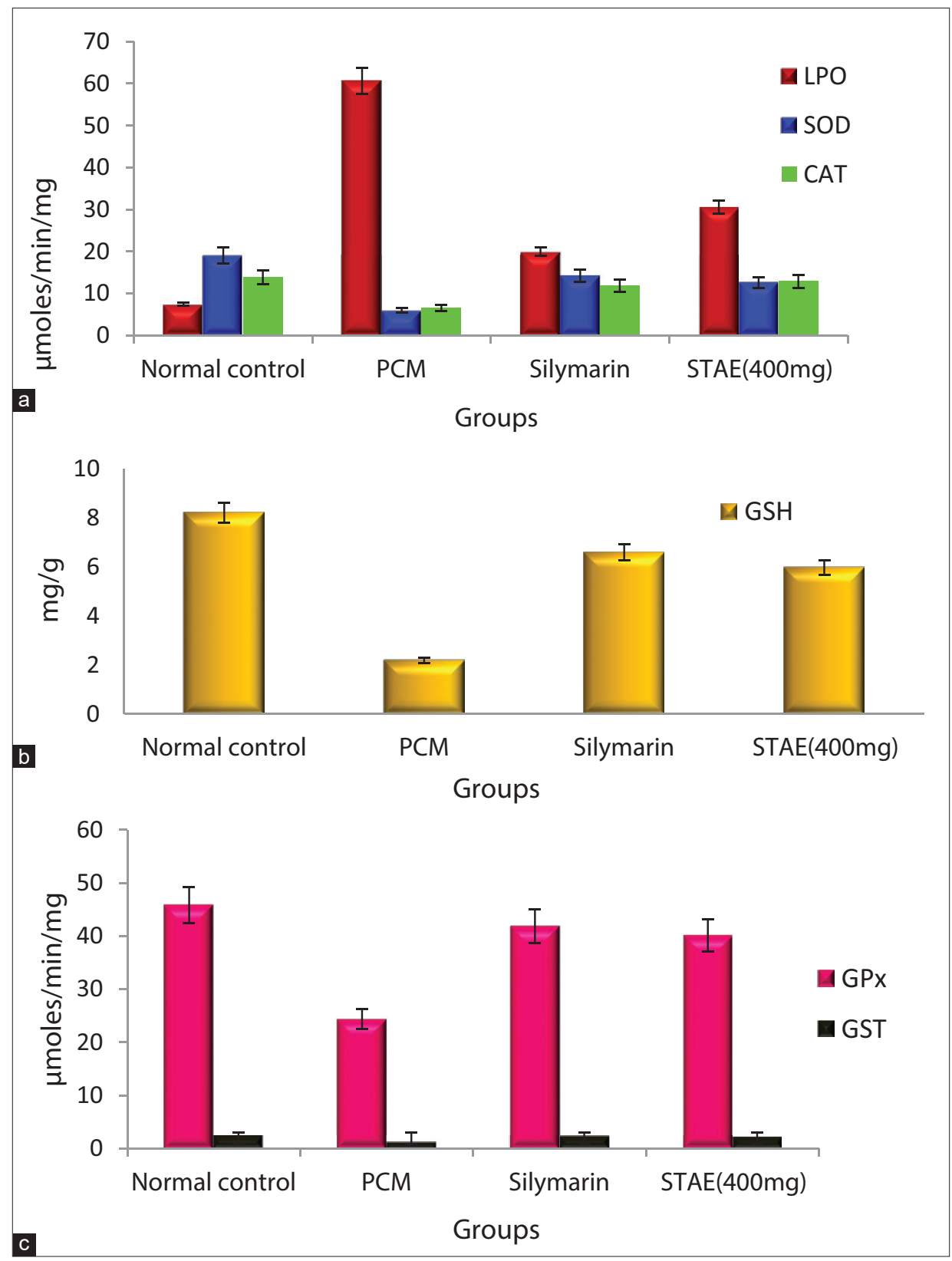

Fig. 1: (a) Effect of aqueous extract of Solanum torvum Sw. on antioxidant status of the experimental animals. (b) Effect of aqueous extract of $S$. torvum Sw. on reduced glutathione level in liver tissue. (c) Effect of aqueous extract of $S$. torvum Sw. on glutathione-dependent enzymes status of the experimental animals

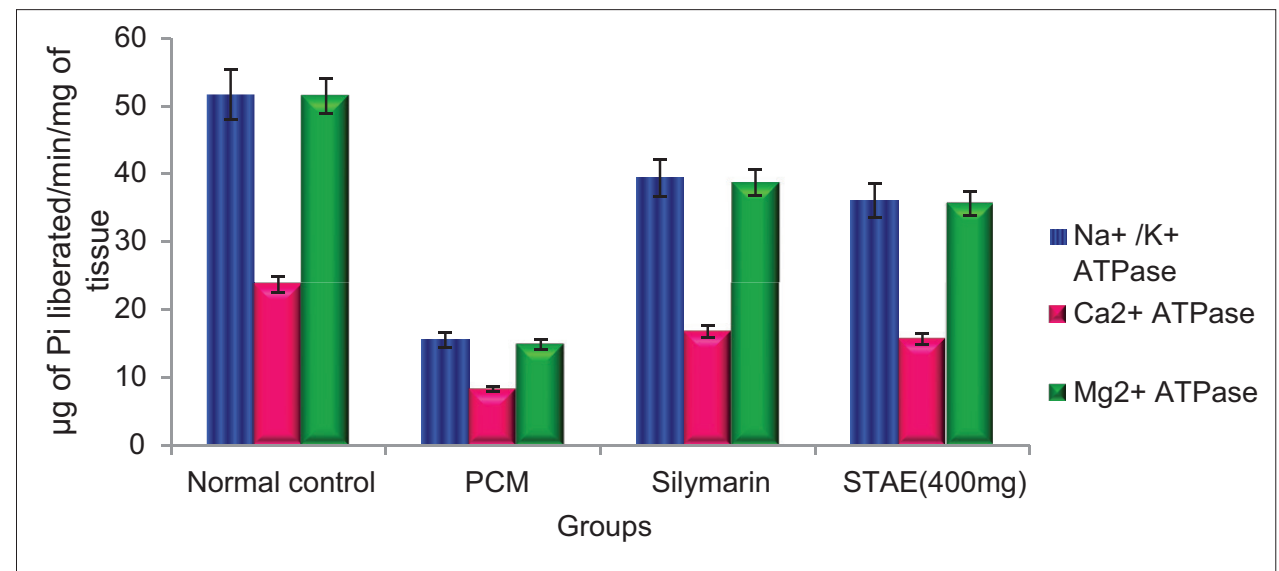

Fig. 2: Effect of aqueous extract of Solanum torvum Sw. on membrane-bound adenosine triphosphatases of liver tissue 


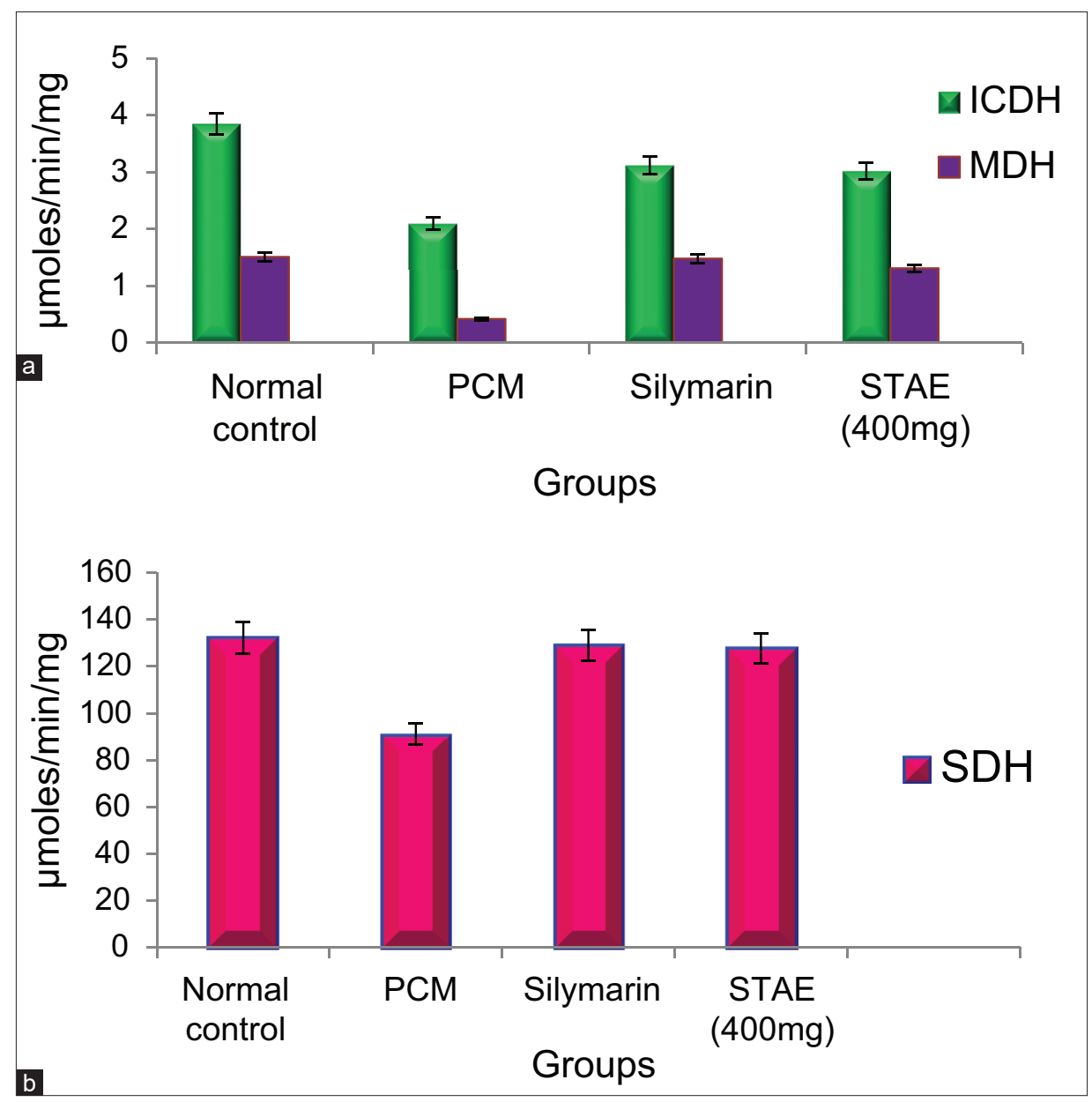

Fig. 3: (a) Effect of aqueous extract of Solanum torvum Sw. on tricarboxylic acid marker enzymes. (b) Effect of aqueous extract of $S$. torvum Sw. on tricarboxylic acid marker enzymes

Inner membrane of mitochondria is particularly at risk from lipid peroxidation because mitochondria utilize oxygen at a high rate and inner membranes have a large content of polyunsaturated fatty acids together with peroxidation catalysts such as iron and copper [14]. In the present study, we found a significant decline in the activities of mitochondrial ICDH, SDH, and MDH in APAP intoxicated rats due to mitochondrial membrane damage caused by lipid peroxidation. Inhibition of the TCA cycle enzymes by ROS may affect the mitochondrial substrate oxidation, resulting in reduced oxidation of substrates, reduced rate of transfer of reducing equivalents to molecular oxygen and depletion of cellular energy [15]. NAPQI directly interacts with sulfhydryl groups on SDH and MDH, causing the loss of its activity [16]. Pre-administration of S. torvum Sw. fruit extract in APAP-treated rats caused a significant elevation in the activities of the TCA cycle enzymes which could be due to the significant uncoupling in mitochondria by the plant extract. A slight uncoupling of oxidative phosphorylation in liver is related to a protective effect against oxidative stress and decreased production of ROS, which is one of the reasons for improved mitochondrial function as well as hepatic protection of the plant extract or probably by preventing the excessive generation of NAPQI with concomitant improvement in the mitochondrial antioxidant defense system, thereby protecting critical nucleophilic sites on the enzymes against toxic electrophilic metabolites [17]. This membrane-stabilizing effect may be attributed to the phytoconstituents such as flavonoids and glycosides present in the extract.

\section{Histopathological studies}

Fig. 4 reveals the histopathological observation of the liver tissue in the control and experimental groups of rats. Histology of the liver sections of normal control rats (Group I) showed normal hepatic cells with well-

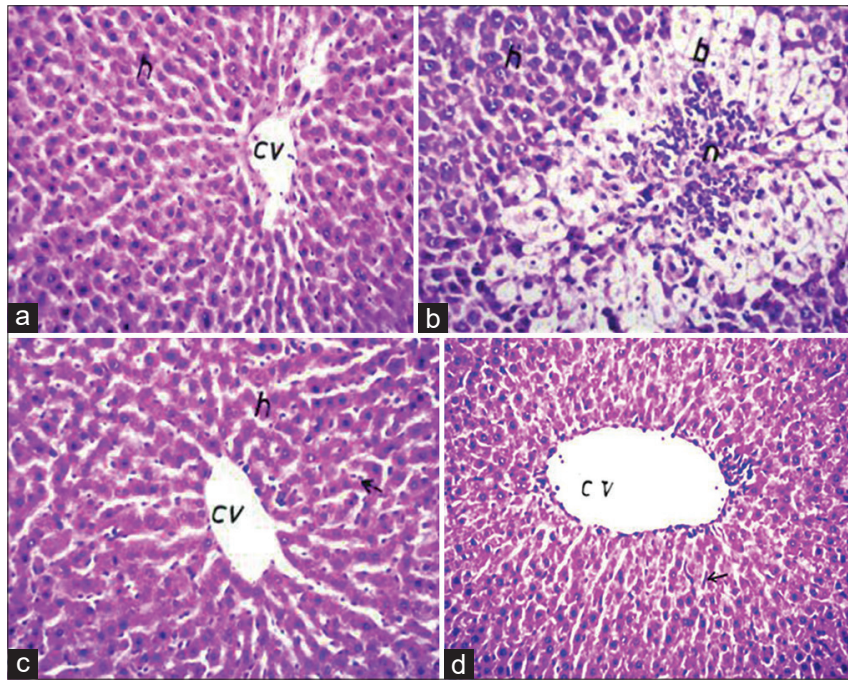

Fig. 4: Histoarchitecture of normal and drug-treated liver tissues.

(a) Control group: Normal histological structure of the liver.

(b) Acetaminophen (APAP)-treated group: Severe focal central vein (CV) and surrounding hepatocytes centrilobular necrosis (n) with central pyknotic nuclei surrounded by ballooning degeneration in most of hepatic parenchyma. (c) Silymarin + APAP-treated group: Dilatation in CV with Kupffer cell proliferation (arrow) between hepatocytes; (d) Solanum torvum aqueous extract ( $400 \mathrm{mg} / \mathrm{kg}$ b.w.) + APAP-treated group: Diffuse. Kupffer cells proliferation between hepatocytes. Hematoxylin-Eosin staining, $\times 40$ 
preserved cytoplasm, prominent nucleus, nucleolus, and visible central veins, whereas that of APAP toxicity-induced rats showed centrilobular necrosis with fatty infiltration leading to hepatocellular degeneration and destruction of architecture in hepatocytes. The group-treated with selected dose of the plant extract showed marked improvement. The cellular arrangement is found to be nearly like the control group exhibiting mild fatty infiltration. It exhibited good regeneration of the hepatocytes and lobular arrangement of the cells. Standard drug silymarin-treated group showed prominent regeneration of the cells with no fatty infiltration and evident necrosis. The study is supportive to the results obtained from the biochemical analysis [18].

\section{CONCLUSION}

The present study is an effort to evaluate the hepatoprotective efficacy of aqueous extract of $S$. torvum Sw. fruit by studying the biochemical and some molecular alteration observed in APAP-induced hepatotoxicity in rats. The in vivo studies revealed that the selected dose of the plant extract was proved to be an effective hepatoprotective agent whose activity was comparable with that of the standard drug silymarin. Histopathological studies revealed centrilobular necrosis in the drug-induced tissues. The plant extract administration repaired the centrilobular necrosis and regeneration of the tissues was noticed, which may be due to the antioxidant effect of the plant extract. An enhancement in LPO levels with a simultaneous depression in the levels of tissue antioxidants and with a decrease in the activities of TCA cycle marker enzymes. It also resulted in membrane damage of the hepatocytes which was evident from the decreased levels of membranebound ATPases activity. S. torvum extract on pre-treatment exerted a significant free radical quenching and antioxidant strengthening effects by decreasing the lipid peroxidation with consequent improvement in antioxidant status proving to be a potent antioxidant and also restored the mitochondrial enzyme activities. Administration of the plant drug also brought back the activity of membrane-bound ATPases indicating the membrane stabilizing potential and antiperoxidative effect of the plant extract. The above results suggested that $S$. torvum Sw. has potential clinical applications. Pre-treatment of $S$. torvum Sw. extract showed hepatoprotection against APAP-induced hepatotoxicity in rats. Antioxidative action has been reported to play an important role in the liver protective ability of many plants. S. torvum Sw. fruit extract may contain antioxidant compounds or a free radical scavenger as it reduces lipid peroxidation and induces antioxidant status. It is known that some flavonoids are able to reduce xenobiotic-induced hepatotoxicity in animals [19]. The inhibitory activity of flavonoids on free radical production could be related to their hepatoprotective effects since exogenous antioxidants may counteract the damaging effects of oxidative stress, cooperating with natural systems such as GSH or protective enzymes.

\section{AUTHORS' CONTRIBUTIONS}

Dr. Umera Begam reporting biochemist prepared the manuscript, Dr. Senthil Kumar supervised and assisted in the preparation of the manuscript, and Dr. Sirajudeen assisted in organizing and reviewing the manuscript.

\section{CONFLICTS OF INTEREST}

The authors declare that they have no conflicts of interest.

\section{REFERENCES}

1. Bernal W, Donaldson N, Wyncoll D, Wendon J. Blood lactate as an early predictor of outcome in paracetamol-induced acute liver failure: A cohort study. Lancet 2002;359:558-63.

2. Moukette BM, Pieme CA, Njimou JR, Biapa CP, Marco B, Ngogang JY. In vitro antioxidant properties, free radicals scavenging activities of extracts and polyphenol composition of a non-timber forest product used as spice: Monodora myristica. Biol Res 2015;48:15.

3. Nguelefacka TB, Catherine BF, Gilber A, Pierre W, Simplice T, Albert DA, et al. Anti-ulcerogenic properties of the aqueous and methanol extracts from the leaves of Solanum torvum Swartz (Solanaceae) in rats. J Ethnopharmacol 2008;119:135-40.

4. Deepak K, Nayak G, Rajalingam D, Sengupta P, Maity K. Evaluation of hepatoprotective and antioxidant activity of Ichnocarpus frutescens (Linn.) R.Br. On paracetamol induced hepatotoxicity in rats. Trop J Pharm Res 2007;6:755-65.

5. Sun F, Hamagawa E, Tsutsui C, Sakaguchi N, Kakuta Y, Tokumaru S, et al. Evaluation of oxidative stress during apoptosis and necrosis caused by D-galactosamine in rat liver. Biochem Pharmacol 2003;65:101-7.

6. Abirami A, Nagarani G, Siddhuraju P. Hepatoprotective effect of leaf extracts from Citrus hystrix and C. Maxima against paracetamol induced liver injury in rats. Food Sci Hum Wellness 2015;4:35-41.

7. Zoubair B, Azzahra LF, Fouzia H, Mohammed L, Brahim B, Noureddine B. Evaluation of acetaminophen effect on oxidative stressed mice by peroxide hydrogen. Am J Biomed Res 2013;1:75-9.

8. Asadi-Samani M, Kafash-Farkhad N, Azimi N, Fasihi A, AliniaAhandani E, Rafieian-Kopa M. Medicinal plants with hepatoprotective activity in Iranian folk medicine. Asian Pac J Trop Biomed 2015;5:146-57.

9. Younes M, Siegers CP. The role of iron in the paracetamol and CCl4induced lipid peroxidation and hepatotoxicity. Chem Biol Interact 1985;55:327-34.

10. Begam AK, Senthilkumar R, Sirajudeen J. J Pharm Pharmacol 2000;52:315.

11. Papadimitrious JC, Phelps PC, Shin ML, Smith MW, Trump BF. Effects of $\mathrm{Ca}^{2+}$ deregulation on mitochondrial membrane potential and cell viability in nucleated cells following lytic complement attack. Cell Calcium 1994; 15:217-27.

12. Hebbel RP, Shalev O, Foker W, Rank BH. Inhibition of erythrocyte $\mathrm{Ca}^{2+}$-ATPase by activated oxygen through thiol-and lipid-dependant mechanisms. Biochem Biophys Acta 1986;862:8-16.

13. Al-Numair KS, Chandramohan G, Alsaif MA, Veeramani C, El Newehy AS. Morin, a flavonoid, on lipid peroxidation and antioxidant status in experimental myocardial ischemic rats. Afr $\mathrm{J}$ Tradit Complement Altern Med 2014;11:14-20.

14. de Hingh YC, Meyer J, Fischer JC, Berger R, Smeitink JA, Op den kamp JA. Direct measurement of lipid peroxidation in submitochondrial particles. Biochemistry 1995;34:12755-60.

15. Capetenaki Y. Desmin cytoskeleton: A potential regulator of muscle mitochondria behavior and function. Trends Cardiovasc Med 2008; 12:339-48.

16. Arathi G, Sachdanandam P. Therapeutic effect of Semecarpus anacardium Linn. Nut milk extract on carbohydrate metabolizing and mitochondrial TCA cycle and respiratory chain enzymes in mammary carcinoma rats. J Pharm Pharmacol 2003;55:1283-90.

17. Sohal RS, Weindruch R. Oxidative stress calorie restriction and aging. Science 1996;273:59-63.

18. Yogi B, Mishra A. Hepatoprotective effects of polyherbal formulation against carbon tetrachloride-induced hepatic injury in albino rats: A toxicity screening approach. Asian J Pharm Clin Res 2017;10:192-5.

19. Saha P, Talukdar AD, DuttaChoudhury M. Antioxidant potential profile of Pajanelia longifolia (willd.) K. Schuman; potential new sources of natural antioxidant. Asian J Pharm Clin Res 2017;10:184-8. 